\title{
CORRIGENDUM
}

\section{Coherent sampling of active mode-locked terahertz quantum cascade lasers and}

frequency synthesis

Stefano Barbieri, Marco Ravaro, Pierre Gellie, Giorgio Santarelli, Christophe Manquest, Carlo Sirtori, Suraj P. Khanna, Edmund H. Linfield \& A. Giles Davies

Nature Photon. 5, 306-313 (2011); published online 24 April 2011; corrected after print 26 April 2011.

In the version of this Article originally published, the $x$-axis scales in Fig. $5 \mathrm{~g}$-i were incorrect. This error has been corrected in the HTML and PDF versions. 